\title{
Queixas técnicas e reações adversas a medicamentos notificadas em um hospital regional no Brasil: um estudo transversal
}

\author{
Technical complaints and adverse drug reactions reported in a regional \\ hospital in Brazil: a cross-sectional study
}

Alan Maicon de Oliveira ${ }^{1}$, Valéria Albuquerque Vaz Rodrigues², Juliana Petini Passerini ${ }^{3}$, Paula Bercelli Zanoveli Pedreiro ${ }^{3}$, Beatriz Alessi Minto²

${ }^{1}$ Faculdade de Ciências Farmacêuticas, Universidade Estadual Paulista (UNESP) - Araraquara (SP), Brasil.

${ }^{2}$ Fundação Educacional de Fernandópolis (FEF) - Fernandópolis (SP), Brasil.

${ }^{3}$ Hospital de Ensino Santa Casa de Fernandópolis (HESCF) - Fernandópolis (SP), Brasil.

DOI: https://dx.doi.org/10.7322/abcshs.v43i1.1015

\section{RESUMO}

Introdução: Reações adversas e os incidentes associados com medicamentos ocasionam a morte e ameaçam a segurança do paciente. No Brasil, persiste-se uma escassez de dados epidemiológicos e uma abordagem ainda insuficiente. Objetivo: Descrever a frequência e as características das notificações de queixas técnicas e reações adversas a medicamentos (RAM) em um hospital regional. Métodos: Estudo transversal, realizado em um hospital de ensino referência para o noroeste do Estado de São Paulo. Por meio do sistema Notivisa da Anvisa, foram contabilizadas as notificações de RAM e queixas técnicas do período de junho de 2012 a julho de 2014. Excluíram-se as não finalizadas e com dados incompletos. Os casos de RAM foram classificados de acordo com o critério de gravidade, sistema de órgãos afetado e caracterização da classe do fármaco suspeito. Ademais, as características do sexo e da idade dos pacientes envolvidos também foram investigadas. Os dados foram descritos como frequência simples e proporções. Resultados: No total, contabilizaram-se 151 (84,8\%) notificações de potenciais RAM e 27 (15,2\%) de queixas técnicas. Mulheres (62,9\%) com idades entre $26-59$ anos (42,4\%) foram predominantes entre os pacientes notificados com RAM. A maioria das reações foram distúrbios da pele $(33,1 \%)$, de gravidade moderada $(70,2 \%)$, provindas de fármacos que atuam no sistema nervoso $(35,8 \%)$. Sobre as queixas técnicas, o extravasamento de líquido do material de acondicionamento foi a ocorrência mais descrita $(40,7 \%)$. Conclusão: As notificações associadas com medicamentos são recorrentes no âmbito hospitalar e as características relatadas embasam conhecimentos sobre o perfil clínico dos episódios adversos apresentados.

Palavras-chave: sistemas de notificação de reações adversas a medicamentos; efeitos colaterais e reações adversas relacionados a medicamentos; epidemiologia descritiva; farmacovigilância; gestão de riscos; segurança do paciente.

\begin{abstract}
Introduction: Adverse reactions and incidents associated with drugs cause death and threaten patient safety. In Brazil, there is still a shortage of epidemiological data on these episodes and an approach still insufficient. Objective: To describe the frequency and characteristics of reports of technical complaints and adverse drug reactions (ADRs) in a regional hospital. Methods: A cross-sectional study was carried out in a Hospital School in the northwest of the São Paulo State. Through the Anvisa's Notivisa system, it was computed the notifications characterized as ADRs and technical complaints from June 2012 to July 2014. The notifications that were not finalized, with status pending completion or with incomplete data, were excluded. The cases of ADRs were classified according to the criterion of severity, organ system affected and characterization of the suspected drug class. The characteristics of the sex and age of the patients involved were also investigated. Data were described as single frequency and proportions. Results: A total of $151(84.8 \%)$ notifications of possible ADRs and 27 (15.2\%) of technical complaints was recorded. Women (62.9\%) aged $26-59$ years $(42.4 \%)$ were predominant among patients reported with ADRs. The majority of the reactions were skin disorders (33.1\%), of moderate severity $(70.2 \%)$, coming from drugs that act on the nervous system (35.8\%). Regarding the technical complaints, the extravasation of liquid from the packaging material was the most described occurrence (40.7\%). Conclusion: The reports associated with drugs are recurrent in the hospital setting and the characteristics reported base knowledge on the clinical profile of the adverse events presented.
\end{abstract}

Keywords: adverse drug reaction reporting systems; drug-related side effects and adverse reactions; epidemiology, descriptive; pharmacovigilance; risk management; patient safety. 


\section{INTRODUÇÃO}

Reações adversas e os incidentes associados com medicamentos são caracterizados por serem responsáveis pela busca por assistência em estabelecimentos de saúde ou, ainda, evoluir para a hospitalização do paciente e até mesmo ocasionar óbito ${ }^{1,2}$. Para tanto, causam impacto negativo nos recursos disponibilizados para serviços de saúde 2 .

Diante de tal agravo, ratifica-se a importância de amplificar conhecimentos sobre os efeitos dos medicamentos e, quando necessário, credenciar condutas de alerta para os mesmos ${ }^{3}$. Assim, preconiza-se a utilidade da vigilância e da disseminação das informações para gerir deliberações cabíveis ${ }^{3}$.

Análises direcionadas para a segurança proporcionada pelo fármaco são realizadas em estudos antes do medicamento ser comercializado, porém, são limitadas ${ }^{4}$. As reações adversas classificadas como raras e muito raras, bem como a ampla ciência do risco-benefício de um medicamento, habitualmente, só são conhecidas na fase pós-comercialização ${ }^{4}$.

Essa falta de referência preliminar e os obstáculos encontrados pelos serviços de vigilância como a subnotificação revelam uma problemática que afeta o paciente ${ }^{5,6}$. Evidência expõe que uma porcentagem média de até $45,11 \%$ é atribuída para episódios evitáveis de reações adversas que originam internações hospitalares ${ }^{7}$.

No Brasil, persiste-se uma escassez de dados epidemiológicos e uma abordagem ainda insuficiente ${ }^{8}$. Há pouca disponibilidade de relatórios com avaliação das reações adversas a medicamentos (RAM) no contexto da assistência à saúde ${ }^{8}$. O objetivo deste estudo é descrever a frequência e as características das notificações de queixas técnicas e RAM em um hospital regional.

\section{MÉTODOS}

\section{Delineamento e contexto}

Trata-se de um estudo transversal descritivo realizado em um hospital de ensino que é referência para 13 munícipios do noroeste do Estado de São Paulo e regiões dos Estados do Mato Grosso, Minas Gerais e Goiás. Dispõe de 174 leitos (16 de terapia intensiva), com média mensal de 640 internações e 360 cirurgias. Destaca-se pelas especialidades no socorro de urgência e emergência, atenção à gestantes de alto risco, assistência de alta complexidade em nefrologia, ortopedia e traumatologia.

Mediante setor de gerenciamento de risco atuante e por meio do portal online da Agência Nacional de Vigilância Sanitária (Anvisa), utiliza-se o Sistema Nacional de Notificações em Vigilância Sanitária (Notivisa) ${ }^{9}$ para registrar as notificações de farmacovigilância ${ }^{10}$. Este banco de dados foi analisado no período de junho de 2012 a julho de 2014.

\section{Critérios de elegibilidade}

Foram elegíveis as notificações de potenciais reações adversas de pacientes internados e queixas técnicas associadas com medicamentos:
- RAM: casos em que a resposta a um medicamento foi prejudicial, não intencional, e ocorreu nas doses normalmente utilizadas em seres humanos ${ }^{11}$;

- Queixa técnica: qualquer irregularidade identificada em relação a um produto, ou ao seu fabricante, e que não foi administrado ao paciente (por ex., desvio da qualidade, produto sem registro ou falsificado $)^{12}$.

Excluíram-se as notificações que não estavam finalizadas, ou seja, com o status pendente de conclusão, assim como as com dados incompletos (referentes ao medicamento suspeito, tipo e evolução da reação e a ocorrência da queixa técnica).

\section{Variáveis e mensuração}

Das notificações de RAM, foram investigadas características referentes aos pacientes, o sexo (masculino e feminino) e a idade (faixa etária (anos): $\leq 25,26-59$ e $\geq 60$ ) foram consideradas. Os episódios foram classificados conforme os seguintes critérios:

- Gravidade: (I) Leve - reação de pouca significância clínica, pequena duração e que não requer intervenções elaboradas; (II) Moderada - reação que interfere nas atividades cotidianas do paciente e que exige alteração na terapêutica e tratamento dos efeitos proporcionados; (III) Grave - reação que ameaça diretamente a vida do paciente, pode ocasionar incapacidade permanente e ser fatal ${ }^{13}$;

- Classificação da reação adversa manifestada: categorização conforme o sistema de órgãos afetado e distribuídas entre os seguintes distúrbios: cardíacos; da audição e labirinto; da pele e tecido subcutâneo; do sangue e sistema linfático; do sistema imunológico; do sistema nervoso; endócrinos, gastrointestinais; hepatobiliares; infecções e infestações; metabólicos e nutricionais; musculoesqueléticos e tecidos conectivos; neoplásicos; no local de administração; psiquiátricos; renais e urinários; reprodutivos; respiratórios e mediastínicos; vasculares; e visuais ${ }^{14}$.

- Caracterização do fármaco suspeito: classificação das classes dos fármacos notificados em concordância com o código ATC (Anatomical Therapeutic Chemical) e mediante aos grupos anatômicos principais: A) Trato alimentar e metabolismo; B) Sangue e órgãos hematopoiéticos; C) Sistema cardiovascular; D) Dermatológicos; G) Sistema geniturinário e hormônios sexuais; H) Hormônios sistêmicos; J) Anti-infecciosos gerais para uso sistêmico; L) Agentes antineoplásicos e imunomoduladores; M) Sistema musculoesquelético; N) Sistema nervoso central; P) Antiparasitários; R) Sistema respiratório; S) Órgãos dos sentidos; e V) Vários ${ }^{15}$.

Determinou-se a causalidade das RAM pelo estudo do caso notificado por meio da melhor evidência disponível na instituição (Micromedex ${ }^{\oplus}$ Solutions. Truven Health Analytics LLC) ${ }^{16}$. Além disso, todas as notificações foram examinadas e discutidas por equipe multidisciplinar e membros da comissão de farmacovigilância do hospital. 
As queixas técnicas foram estratificadas de acordo com os tipos de ocorrências reportados.

\section{Métodos estatísticos}

Procedeu-se à estatística descritiva das variáveis mensuradas. Os dados foram estruturados no Programa Microsoft Office Excel $^{\oplus}$ versão 2016, quantificados e utilizados em tabulação. Foram descritos como frequência simples e proporções.

\section{Aspectos éticos}

Este estudo teve o consentimento da Comissão de Ensino e Pesquisa do Hospital, foi submetido à Plataforma Brasil e aprovado de acordo com o parecer $n^{\circ} 1.201 .825$.

\section{RESULTADOS}

No período estudado, foram contabilizadas 178 notificações associadas a medicamentos. Este total fragmentou-se em 151 $(84,8 \%)$ notificações de potenciais RAM e $27(15,2 \%)$ de queixas técnicas (Figura 1). Não houve notificações com os dados considerados incompletos.

A representação da gravidade dos casos de RAM foi descrita de acordo com o sexo e a faixa etária dos pacientes (Tabela 1). Percebe-se que 27 (17,9\%) casos foram considerados leves, 106 $(70,2 \%)$ moderados e $18(11,9)$ graves. Ao todo, 95 (62,9\%) episódios ocorreram em mulheres e $56(37,1 \%)$ em homens. A faixa etária de 26-59 anos foi a mais notificada ( $\mathrm{n}=64 ; 42,4 \%)$. Contudo, em relação ao total de reações graves, indivíduos com 60 anos ou mais foram os que mais sofreram estes episódios ( $n=10 ; 55,6 \%)$ e, dentre estes, destacou-se um óbito em paciente diagnosticado com a síndrome de Stevens Johnson.

A Tabela 2 mostra a frequência dos tipos de reações que se manifestaram nos pacientes conforme o sistema de órgãos afetado. Constatou-se que os distúrbios da pele e tecido subcutâneo foram os casos mais apresentados $(n=50 ; 33,1 \%)$. Por conseguinte,

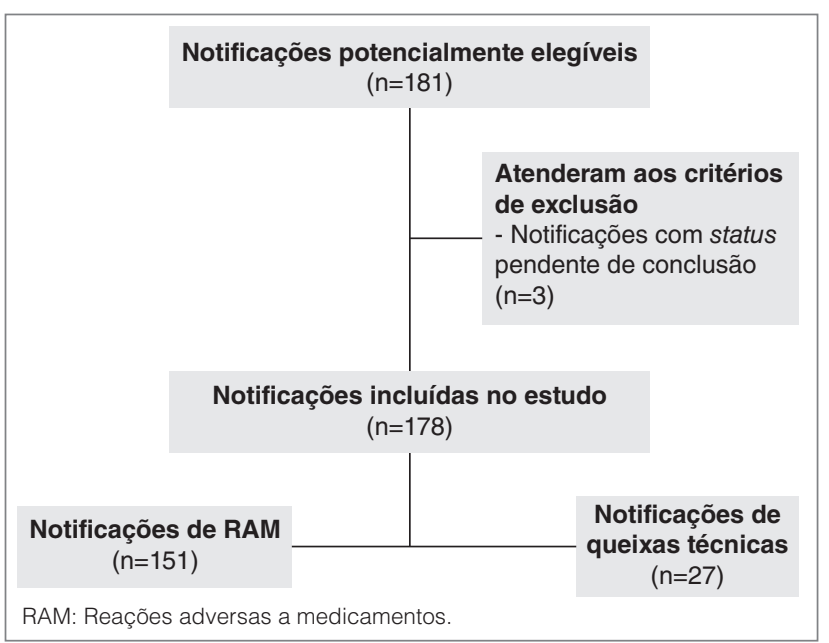

Figura 1: Fluxograma da seleção das notificações reportaram-se os distúrbios gastrointestinais $(n=20 ; 13,2 \%)$ e no local da administração ( $\mathrm{n}=17 ; 11,3 \%)$.

$\mathrm{Na}$ maioria das notificações, os fármacos mais relatados, em concordância com a classe ATC, foram os que atuam no sistema nervoso ( $\mathrm{n}=54 ; 35,8 \%) .38$ (25,2\%) episódios estavam associados com a classe dos anti-infecciosos gerais para uso sistêmico e 32 $(21,2 \%)$ com os fármacos que agem no trato alimentar e no metabolismo (Tabela 3).

Em relação às notificações de queixas técnicas, averiguou-se que o tipo de ocorrência que se sobressaiu foi o extravasamento de líquido do material de acondicionamento $(\mathrm{n}=11 ; 40,7 \%)$, seguido pela mudança de coloração do produto $(\mathrm{n}=5 ; 18,5 \%)$ (Tabela 4$)$.

Tabela 1: Distribuição das notificações de potenciais reações adversas a medicamentos por sexo, idade e gravidade. SP, Brasil, 2012-2014. $(n=151)$

\begin{tabular}{|c|c|c|c|c|}
\hline \multirow{2}{*}{$\begin{array}{c}\text { Características } \\
\text { Sexo } \\
\text { Masculino }\end{array}$} & Leve & $\begin{array}{c}\text { RAM notificadas } \\
\text { Moderada }\end{array}$ & Grave & Total (\%) \\
\hline $\begin{array}{c}\text { Feminino } \\
\text { Idade (anos) }\end{array}$ & 21 & 43 & 7 & $56(37,1)$ \\
\hline$\leq 25$ & & 63 & 11 & $95(62,9)$ \\
\hline $26-59$ & 11 & 48 & 5 & $64(42,4)$ \\
\hline$\geq 60$ & 13 & 33 & 10 & $56(37,1)$ \\
\hline Total (\%) & $27(17,9)$ & $106(70,2)$ & $18(11,9)$ & \\
\hline
\end{tabular}

RAM: Reações adversas a medicamentos.

Tabela 2: Distribuição da frequência das reações adversas a medicamentos de acordo com o sistema de órgãos afetado. SP, Brasil, 2012-2014. $(n=151)$

\begin{tabular}{|l|c|c|}
\hline Sistema de órgãos & Frequência & $\%$ \\
\hline Distúrbios da pele e tecido subcutâneo & 50 & 33,1 \\
\hline Distúrbios gastrointestinais & 20 & 13,2 \\
\hline Distúrbios no local de administração & 17 & 11,3 \\
\hline Distúrbios cardíacos & 16 & 10,6 \\
\hline Distúrbios do sistema nervoso & 14 & 9,3 \\
\hline Distúrbios psiquiátricos & 14 & 9,3 \\
\hline Distúrbios respiratórios, torácicos e mediastínicos & 9 & 6 \\
\hline Distúrbios do sistema imunológico & 6 & 4 \\
\hline Distúrbios vasculares & 3 & 2 \\
\hline Distúrbios musculoesqueléticos e tecidos & 2 & 1,3 \\
\hline conectivos & & \\
\hline
\end{tabular}

Tabela 3: Classificação dos fármacos notificados suspeitos de causarem as reações adversas. SP, Brasil, 2012-2014. ( $n=151)$

\begin{tabular}{|l|c|c|}
\hline Classe ATC & Frequência & $\%$ \\
\hline [N] Sistema nervoso & 54 & 35,8 \\
\hline [J] Anti-infecciosos gerais para uso sistêmico & 38 & 25,2 \\
\hline [A] Trato alimentar e metabolismo & 32 & 21,2 \\
\hline [R] Sistema respiratório & 8 & 5,3 \\
\hline [M] Sistema musculoesquelético & 7 & 4,6 \\
\hline [B] Sangue e órgãos hematopoiéticos & 6 & 4 \\
\hline [C] Sistema cardiovascular & 4 & 2,6 \\
\hline [L] Agentes antineoplásicos e imunomoduladores & 1 & 0,7 \\
\hline [D] Dermatológicos & 1 & 0,7 \\
\hline
\end{tabular}

ATC: Anatomical Therapeutic Chemical. 


\section{DISCUSSÃO}

No hospital analisado, prevaleceram as notificações de RAM. Mulheres, com idades entre 26-59 anos, foram às características predominantes nos pacientes informados. A maioria das RAM foi classificada como distúrbios da pele, de gravidade moderada, provinda de fármacos que atuam no sistema nervoso. Em relação às queixas técnicas, o extravasamento de líquido do material de acondicionamento foi o mais relatado.

Enfatiza-se que a equipe multidisciplinar teve acesso aos relatórios de reações adversas durante o período de internação dos pacientes e, com isso, obteve a possibilidade de intervenções. As notificações de queixas técnicas relatavam casos de desvios de qualidade dos medicamentos e, se houvesse contato do paciente com esses produtos, poderiam ocorrer danos à saúde. Contemplase, assim, o benefício resultante para segurança do paciente.

Pesquisas que analisaram notificações associadas a medicamentos detectaram, em média, frequência de relatos de RAM semelhante ao presente estudo (variou entre 90 a 118 por ano $)^{17,18}$. O monitoramento dos efeitos dos medicamentos, assim como dos incidentes proporcionados, é de extrema necessidade ${ }^{3}$. Incentivos aos profissionais da saúde e capacitações para a população em geral, a fim de promover a notificação espontânea, tornaram-se primordiais para este cenário ${ }^{4}$. Assim, com a educação em saúde, pretende-se que as RAM possam ser evitadas e não só relatas após o seu acontecimento ${ }^{3}$.

Outros pesquisadores também identificaram maior proporção de pacientes do sexo feminino que sofreram RAM $^{5,19}$. Esta susceptibilidade das mulheres é explicada, possivelmente, devido às condições obstétricas, bem como alterações metabólicas pertinentes ao desequilíbrio hormonal ${ }^{20}$.

Pesquisa realizada em um hospital no Brasil identificou maiores taxas de RAM em pacientes adultos (19-59 anos) ${ }^{8}$, dados

Tabela 4: Tipo de ocorrência das queixas técnicas de medicamentos notificadas. SP, Brasil, 2012-2014. ( $n=27)$

\begin{tabular}{|l|c|c|}
\hline Queixa técnica & Frequência & $\%$ \\
\hline Extravasamento de líquido do material de & 11 & 40,7 \\
\hline acondicionamento & 5 & 18,5 \\
\hline Mudança de coloração & 4 & 14,8 \\
\hline Presença de partículas estranhas & 4 & 14,8 \\
\hline Material de acondicionamento danificado & 3 & 11,1 \\
\hline Precipitação
\end{tabular}

concordantes com o nosso estudo. Evidências apoiam que a idade avançada é fator de risco para as RAM devido as modificações farmacodinâmicas e farmacocinéticas ${ }^{19,20}$.

Estudos que avaliaram a gravidade dos casos de RAM também apresentaram resultados semelhantes a esta pesquisa, com prevalência dos episódios moderados e leves ${ }^{8,17,21}$. Devido ao ato de notificar, que proporciona uma maior atenção para a reação exposta, ocorrem as intervenções e, por isso, existe menos risco de evolução para um caso grave ${ }^{8}$.

Outros autores também detectaram os distúrbios da pele e gastrointestinais entre os mais frequentes das reações manifestadas $^{8,20-22}$. Uma evidência ainda destaca os distúrbios no local de administração ${ }^{22}$ e algumas ressaltam os transtornos do sistema nervoso $^{8,20-22}$, assim como os renais e urinários ${ }^{20}$.

Estudos que identificaram os medicamentos potencialmente causadores das reações adversas, mostraram que os fármacos que agem no sistema nervoso e os anti-infecciosos foram os mais comumente relatados ${ }^{8,22}$, achados concordantes com a nossa pesquisa. Além dessas classes, os fármacos que atuam no sistema cardiovascular ${ }^{17}$ e os agentes antineoplásicos e imunomoduladores ${ }^{21}$ também são notórios nos estudos.

Em relação às queixas técnicas de medicamentos, uma pesquisa realizada em um hospital sentinela no Brasil constatou que os vazamentos e a coloração diferente do habitual foram os tipos de ocorrências mais notificados ${ }^{23}$. Portanto, enfatiza-se o resultado parecido.

Como uma limitação do estudo atual, observa-se que não foi utilizado um método validado específico para determinar a causalidade das potenciais reações apresentadas pelos pacientes. Todavia, conforme já descrito anteriormente, profissionais especializados e a melhor evidência disponível foram consultados para estudo dos casos notificados.

Em conclusão, as notificações de queixas técnicas e RAM mostraram-se recorrentes no contexto hospitalar. Outrossim, amparam intervenções importantes para a segurança dos pacientes. As características das notificações embasam conhecimentos robustos sobre os efeitos dos medicamentos e referentes ao perfil clínico dos episódios adversos apresentados.

\section{AGRADECIMENTOS}

Ao Núcleo Hospitalar de Epidemiologia e à Comissão de Segurança do Paciente do Hospital de Ensino Santa Casa de Fernandópolis.

\section{REFERÊNCIAS}

1. Zed PJ, Haughn C, Black KJL, Fitzpatrick EA, Ackroyd-Stolarz $\mathrm{S}$, Murphy NG, et al. Medication-related emergency department visits and hospital admissions in pediatric patients: a qualitative systematic review. J Pediatr. 2013;163(2):477-83. https://dx.doi.org/10.1016/j.jpeds.2013.01.042

2. Freitas GRM, Tramontina MY, Balbinotto G, Hughes DA, Heineck I. Economic impact of emergency visits due to drug-related morbidity on a Brazilian hospital. Value Health Reg Issues. 2017;14(Suppl C):1-8. https://dx.doi.org/10.1016/j.vhri.2017.03.003
3. Laporte J-R. Fifty years of pharmacovigilance: medicines safety and public health. Pharmacoepidemiol Drug Saf. 2016;25(6):725-32.

https://dx.doi.org/10.1002/pds.3967

4. Santoro A, Genov G, Spooner A, Raine J, Arlett P. Promoting and protecting Public Health: How the European Union Pharmacovigilance System Works. Drug Saf. 2017;40(10):855-69. https://dx.doi.org/10.1007/s40264-017-0572-8 
5. Rydberg DM, Holm L, Engqvist I, Fryckstedt J, Lindh JD, Stiller $\mathrm{CO}$, et al. Adverse drug reactions in a tertiary care emergency medicine ward - prevalence, preventability and reporting. PLoS One. 2016;11(9):e0162948.

https://dx.doi.org/10.1371/journal.pone.0162948

6. Alatawi YM, Hansen RA. Empirical estimation of under-reporting in the U.S. Food and Drug Administration Adverse Event Reporting System (FAERS). Expert Opin Drug Saf. 2017;16(7):761-7. http://dx.doi.org/10.1080/14740338.2017.1323867

7. Patel NS, Patel TK, Patel PB, Naik VN, Tripathi C. Hospitalizations due to preventable adverse reactions-a systematic review. Eur J Clin Pharmacol. 2017;73(4):385-98.

https://dx.doi.org/10.1007/s00228-016-2170-6

8. Lobo MGAA, Pinheiro SMB, Castro JGD, Momenté VG, Pranchevicius MCS. Adverse drug reaction monitoring: support for pharmacovigilance at a tertiary care hospital in Northern Brazil. BMC Pharmacol Toxicol. 2013;14:5. https://dx.doi.org/10.1186/2050-6511-14-5

9. Agência Nacional de Vigilância Sanitária (ANVISA). Sistema de Notificações para a Vigilância Sanitária (Notivisa). Disponível em: http://portal.anvisa.gov.br/notivisa Acesso em: 03 jan. 2017.

10. World Health Organization (WHO). The importance of pharmacovigilance: safety monitoring of medicinal products. Geneva: World Health Organization; 2002.

11. World Health Organization (WHO). International drug monitoring: the role of the hospital. Geneva: World Health Organization; 1966.

12. Agência Nacional de Vigilância Sanitária (ANVISA). Sistema Nacional de Notificações para a Vigilância Sanitária. Manual do Usuário: formulário para notificação de eventos adversos à medicamento. Disponível em: http://www.anvisa.gov.br/hotsite/ notivisa/manual/ea_medicamento_profissional.pdf. Acesso em: 04 ago. 2017.

13. Hartwig SC, Siegel J, Schneider PJ. Preventability and severity assessment in reporting adverse drug reactions. Am J Hosp Pharm. 1992;49(9):2229-32.

14. National Institutes of Health. National Cancer Institute. Common Terminology Criteria for Adverse Events (CTCAE). US Department of Health and Human Services. 2009;4(3):1-78.
15. World Health Organization (WHO). Collaborating Centre for Drug Statistics Methodology, Guidelines for ATC classification and DDD assignment 2013. Oslo, 2012. Disponível em: https://www.whocc.no/ filearchive/publications/1_2013guidelines.pdf/Acesso em: 21 set. 2017.

16. Truven Health Analytics. Micromedex® Solutions. Disponivel em: http://www.micromedexsolutions.com/home/dispatch. Acesso em: 21 set. 2017.

17. Mendes D, Alves C, Marques FB. latrogenia grave desconhecida, notificações e notificadores: resultados da actividade da Unidade de Farmacovigilância do Centro. Rev Port Med Geral Fam. 2012;28(1):34-40

18. Chen CJ, Cheng CF, Lin HY, Hung SP, Chen WC, Lin MS. A comprehensive 4-year survey of adverse drug reactions using a network-based hospital system. J Clin Pharm Ther. 2012;37(6):647-51. https://dx.doi.org/10.1111/j.1365-2710.2012.01359.x

19. Ruiter R, Visser LE, Rodenburg EM, Trifiró G, Ziere G, Stricker BH. Adverse drug reaction-related hospitalizations in persons aged 55 years and over: a population-based study in the Netherlands. Drugs Aging. 2012;29(3):225-32. https://dx.doi.org/10.2165/11599430-000000000-00000

20. Pedrós C, Quintana B, Rebolledo M, Porta N, Vallano A, Arnau $\mathrm{JM}$. Prevalence, risk factors and main features of adverse drug reactions leading to hospital admission. Eur J Clin Pharmacol. 2014;70(3):361-7

https://dx.doi.org/10.1007/s00228-013-1630-5

21. Kim B, Kim SZ, Lee J, Jung AH, Jung SH, Hahn HJ, et al. Clinical profiles of adverse drug reactions spontaneously reported at a single Korean hospital dedicated to children with complex chronic conditions. PLoS One. 2017;12(2): e0172425.

https://dx.doi.org/ 10.1371/journal.pone.0172425

22. Rosli R, Ming LC, Abd Aziz N, Manan MM. A retrospective analysis of spontaneous adverse drug reactions reports relating to paediatric patients. PLoS One. 2016;11(6):e0155385. https://dx.doi.org/10.1371/journal.pone.0155385

23. Lima PFd, Cavassini ACM, Silva FAT, Kron MR, Gonçalves SF, Spadotto A, et al. Queixas técnicas e eventos adversos a medicamentos notificados em um hospital sentinela do interior de São Paulo, 2009-2010. Epidemiol Serv Saúde. 2013;22(4):679-86. http://dx.doi.org/10.5123/S1679-49742013000400014 